\title{
Lung cancer management during the COVID-19 pandemic: experience of a medical oncology unit at a tertiary hospital in Singapore
}

\author{
Elise KY Vong ${ }^{1,2, \#, ~ P u e y-L i n g ~ C h i a ~}{ }^{1,2, \#}$, Alex Y. Chang ${ }^{1,3}$ \\ 'Department of Medical Oncology, Tan Tock Seng Hospital, Singapore. \\ ${ }^{2}$ Lee Kong Chian School of Medicine, Singapore. \\ 3 Johns Hopkins University School of Medicine, Baltimore, MD 21836, USA. \\ \#Authors contributed equally.
}

Correspondence to: Dr Elise KY Vong, Department of Medical Oncology, Tan Tock Seng Hospital, 11 Jalan Tan Tock Seng, Singapore 308433, Singapore. E-mail: elise_ky_vong@ttsh.com.sg

\begin{abstract}
How to cite this article: Vong EKY, Chia PL, Chang AY. Lung cancer management during the COVID-19 pandemic: experience of a medical oncology unit at a tertiary hospital in Singapore. J Cancer Metastasis Treat 2020;6:54.

http://dx.doi.org/10.20517/2394-4722.2020.116
\end{abstract}

Received: 22 Oct 2020 First Decision: 16 Nov 2020 Revised: 30 Nov 2020 Accepted: 2 Dec 2020 Published: 24 Dec 2020

Academic Editor: Robert Kratzke Copy Editor: Monica Wang Production Editor: Jing Yu

\begin{abstract}
Coronavirus disease 2019 (COVID-19), caused by the severe acute respiratory syndrome coronavirus 2 (SARSCoV-2), was declared a pandemic by the World Health Organisation (WHO) on 11 March 2020. The pandemic has had profound effects on healthcare systems across the world, and also poses unique challenges for oncology services. Singapore saw its first imported case of COVID-19 on 23 January 2020, and there has since been 52,000 confirmed cases and 27 deaths as of early August 2020 locally. Oncologists have a special duty to our patients to ensure patient safety and provide optimum care without undue disruption which may compromise long-term cancer-specific outcomes. We herein examine the impact that the COVID-19 pandemic has had on our clinical services, and share our experience with regards to manpower reconfiguration, infection control measures, diagnostic evaluation of patients with suspected COVID-19, oncological management of lung cancer patients, as well as changes in the education and training of juniors, from our unique position as a Medical Oncology department in Tan Tock Seng Hospital, a tertiary hospital affiliated with the National Centre of Infectious Diseases in Singapore.
\end{abstract}

Keywords: COVID-19, lung cancer, thoracic cancer

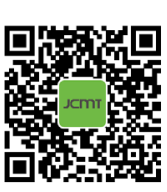




\section{INTRODUCTION}

Since the identification of a cluster of novel viral pneumonia cases caused by the severe acute respiratory syndrome coronavirus 2 (SARS-CoV-2) that was first reported in Wuhan, China, in December 2019, coronavirus disease 2019 (COVID-19) has spread rapidly across the world. As of early August 2020, global cases of COVID-19 have exceeded 18 million, with close to 700,000 deaths and case fatality ratios (CFRs) ranging from $0.1 \%$ to $15 \%$ across different countries ${ }^{[1]}$. Singapore saw its first imported case on 23 January 2020, and had one of the highest numbers of reported COVID-19 cases outside of China in February 2020. There has since been 52,000 confirmed cases and 27 deaths as of early August 2020 locally. Early supportive care and monitoring are the cornerstones of clinical management for the majority of patients with mild illness. In patients with severe illness, dexamethasone and remdesivir are 2 drugs that have been shown in randomised phase 3 trials thus far to reduce mortality ${ }^{[2]}$ and improve time to recovery ${ }^{[3]}$, respectively.

Building on past experience with the severe acute respiratory syndrome (SARS) outbreak in 2003, Singapore has systemically strengthened its ability to manage emerging infectious diseases outbreaks. Since 7 February 2020, Singapore has been in Disease Outbreak Response System Condition (DORSCON) Orange, the second highest national alert level signifying that SARS-CoV-2 has not spread widely in Singapore and is still being contained ${ }^{[4]}$. A multi-agency taskforce was formed to coordinate the national response to the outbreak. National public health measures implemented include aggressive contact tracing, mandatory stay home notices for returning travellers, travel restrictions, telecommuting, mandatory maskwearing and safe distancing measures at public spaces, workplaces and schools.

The National Centre for Infectious Diseases (NCID) is a 330-bed purpose-built facility officially opened in September 2019, designed to augment Singapore's capabilities in infectious disease management and prevention $^{[5]}$. It has been the epicentre of the national response to COVID-19. The provision of clinical services at NCID is supported by the National Healthcare Group (NHG) and Tan Tock Seng Hospital (TTSH), which is situated next to NCID within the Novena campus located in central Singapore. TTSH is Singapore's second largest tertiary care hospital with over 1,700 beds. The medical oncology department at TTSH is the third largest oncology provider amongst tertiary public hospitals in Singapore, and also works closely with other clinical departments and services in TTSH.

The COVID-19 pandemic poses several unique challenges for oncology services, and in particular the management of patients with thoracic malignancies. A key challenge faced by oncologists is the balance of the risk of increased severity of COVID-19 in lung cancer patients versus the consequences of delay in diagnosis and time-sensitive oncological treatment which may occur due to reasons such as changes in health-seeking behaviour and reallocation of healthcare resources to COVID-19. A global survey of medical oncologists has demonstrated that COVID-19 has significantly influenced decision-making by oncologists and significant reductions in cancer-related patient encounters have also been reported ${ }^{[6-8]}$. We herein describe several key experiences relevant to the field of thoracic oncology during the COVID-19 pandemic from the perspective of our department and as guided by available data in the literature.

\section{FACILITY AND MANPOWER SEPARATION AND CROSS-DEPARTMENTAL COLLABORATION}

COVID-19 is spread primarily via droplets and is highly infectious with a basic reproduction number $\left(\mathrm{R}_{0}\right)$ of 2.0-2.5, with established community transmission that has threatened to overwhelm healthcare systems globally ${ }^{[9]}$. Disease severity ranges from mild illness in the majority (80\%) of patients to severe illness requiring mechanical ventilation and intensive care ${ }^{[9]}$. Patients with advanced age and comorbidities including cancer, chronic respiratory disease (COPD), uncontrolled hypertension and diabetes are at increased risk of severe illness and mortality from COVID- $19^{[10]}$. Infection with SARS-CoV-2 can cause considerable disruption to oncological treatment plans. These factors underscore the critical need for 
a coordinated effort to ensure operational continuity with minimal disruption to cancer care while maintaining patient and staff safety.

\section{Infection control measures and general patient management algorithms}

Infection control measures were ramped up rapidly at NCID and TTSH to ensure that all staff are adequately protected from infection risk. NCID and TTSH wards were segregated into wards for nonCOVID-19, suspected and confirmed COVID-19 patients. Patients presenting with acute respiratory infection (ARI) to the NCID Screening Centre (SC) or TTSH Emergency Department (ED) were identified and separated from the general patient pool at the point of triage in order to reduce the risk of exposure to other patients and healthcare staff. At subsequent medical assessment, SARS-CoV-2 reverse transcriptionpolymerase chain reaction (RT-PCR) testing as well as laboratory and chest radiography were conducted according to hospital protocols.

Suspect and confirmed COVID-19 patients were admitted to negative-pressure isolation rooms in NCID for further management. Patients diagnosed with community-acquired pneumonia were admitted to neutral pressure isolation rooms in TTSH under an enhanced pneumonia surveillance program and were required to undergo SARS-CoV-2 testing before they could be de-isolated. The result of SARS-CoV-2 RTPCR testing is generally available in less than 6 hours when performed in NCID or TTSH, and this quick turnaround time has been an important factor in the success and efficiency of the local screening and containment measures. Suspect case definitions for COVID-19 were updated regularly based on evolving epidemiological factors and disseminated by the Ministry of Health $(\mathrm{MOH})$ to all doctors via email, and patient management algorithms were updated accordingly by hospital management.

Other key measures undertaken include compulsory personal protective equipment (PPE) training and N95 mask-fitting for all healthcare workers. Strict adherence to hospital PPE protocols is mandated when reviewing patients who are suspect or confirmed cases of COVID-19. For routine patient care, surgical mask and meticulous hand hygiene is practiced. All TTSH staff were provided with personal thermometers for twice-daily online temperature recording. Staff with ARI were advised to seek medical attention promptly at NCID or the TTSH Occupational Health Clinic for assessment and COVID-19 diagnostic testing, and were provided at least 5 days of medical leave.

TTSH also put in place temperature screening and heath declaration measures for patients and visitors at hospital entrances. Measures for physical distancing were implemented in our outpatient oncology clinics and chemotherapy units. Patients attending outpatient appointments were limited to one accompanying person at any one time. Designated consult rooms and PPE are employed for the assessment of patients with ARI before they are appropriately redirected to NCID or the TTSH ED [Figure 1]. For patients who are on quarantine orders due to close contact with confirmed COVID-19 cases or those on stay home notices after return from abroad, non-urgent outpatient appointments were postponed until after completion of the quarantine orders.

\section{Clinical service reconfiguration}

The COVID-19 pandemic has also led to service reconfiguration of our medical oncology department. One of the first measures undertaken by our department during the start of the outbreak in Singapore was team segregation, in order to reduce the loss of workforce and avoid quarantine of the whole department in the event of COVID-19 exposure or infection. We segregated our department into 2 independently functioning teams each comprising junior doctors, fellows and attendings geographically confined to the inpatient and outpatient sectors. A similar approach has been reported by other cancer centres in Singapore as well ${ }^{[11,12]}$. Large group gatherings were minimised; department meetings and education activities were conducted in small groups and via secure video-conferencing platforms. 


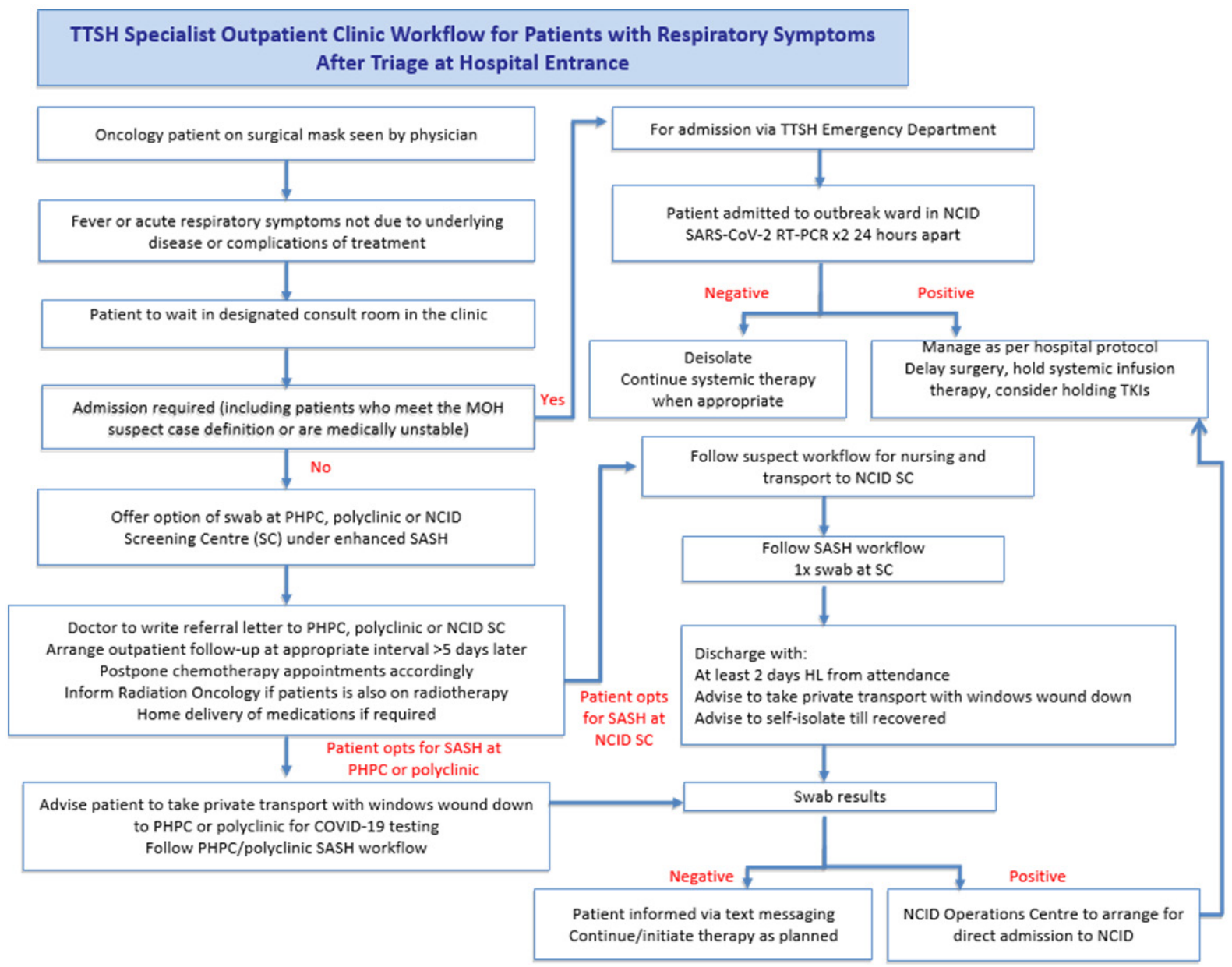

Figure 1. TTSH specialist outpatient clinic workflow for patients with respiratory symptoms after triage at hospital entrance. TTSH: Tan Tock Seng Hospital; MOH: Ministry of Health; NCID: The National Centre for Infectious Diseases; SASH: Swab-and-Send-Home; PHPC: Public Health Preparedness Clinic; SC: screening Centre; COVID-19: Coronavirus disease 2019; RT-PCR: reverse transcriptionpolymerase chain reaction; TKIs: tyrosine kinase inhibitors; SARS-CoV-2: severe acute respiratory syndrome virus 2

Physicians' non-essential leave was also cancelled in order to maximise existing manpower resources. The Singapore $\mathrm{MOH}$ also prohibited cross-hospital movement of physicians, thus necessitating clinic closures and redistribution of patients under the care of our visiting medical oncology consultants. Similarly, interhospital transfers of inpatients were discouraged unless there is a clinical need for specialised medical services not available at the parent hospital. Medical oncologists have also had to take up different duties in the management of the COVID-19 pandemic, with clinical redeployment to NCID on a regular rotational basis to provide acute care for confirmed and suspect COVID-19 patients as part of pandemic coverage in a broader hospital and national effort.

\section{COVID-19 SCREENING IN LUNG CANCER PATIENTS}

A recent meta-analysis has reported a pooled prevalence of cancer in patients with COVID-19 of 2.0\%, an increased incidence compared to the general population ${ }^{[13]}$. Multicentre registries and observational studies have demonstrated an association between increased COVID-19 mortality of 13\%-28\% in patients with cancer and COVID-19 ${ }^{[14,15]}$. Common independent factors include active cancer (as opposed to cancer in remission), underlying comorbidities such as COPD, smoking status, advanced age, and male gender ${ }^{[14,15]}$. Patients with Eastern Cooperative Oncology Group (ECOG) performance status of 2 or higher are at increased risk of worse outcomes from COVID- $19^{[14]}$. One study has demonstrated lower rates of SARS- 
CoV-2 antibody production (seroconversion) in cancer versus non-cancer patients after symptomatic COVID- $19^{[16]}$.

Patients with lung cancers are thought to be at disproportionately increased risk of death from COVID-19, with mortality ranging from $25 \%-33 \%{ }^{[17]}$. However, the increase in mortality is thought to be related to patient-specific features as outlined above, rather than cancer-specific features or specific anti-cancer treatments such as tyrosine kinase inhibitors (TKIs), cytotoxic chemotherapy, or immune checkpoint inhibitors (ICIs), albeit compared to cancer instead of non-cancer controls. The ongoing TERAVOLT study is the largest cohort study of patients with thoracic malignancies including non-small cell lung cancer (NSCLC), small cell lung cancer (SCLC), mesothelioma, thymic epithelial tumours and other pulmonary neuroendocrine neoplasms ${ }^{[17]}$. Preliminary results have been reported from the first 200 patients enrolled, of whom 151 (76\%) had NSCLC, 29 (15\%) had SCLC, $36(18 \%)$ and $147(74 \%)$ had stage III and stage IV disease respectively, and 147 (74\%) were on active anticancer therapy. Forty-eight (33\%), 34 (23\%), and $28(19 \%)$ were on chemotherapy, ICIs or TKIs alone respectively, and 20 (14\%) were on chemotherapy in combination with ICIs. The majority of patients received therapy administered for a median of 7 days (interquartile range 0-17) before COVID-19 diagnosis. Eighty percent of patients developed pneumonia or pneumonitis, and $27 \%$ of patients developed acute respiratory distress syndrome. The mortality rate was $33 \%$. In multivariable analysis, only smoking was significantly associated with an increased risk of death. Although the findings above are highly relevant and have allowed quick dissemination of real-time data and guidance in the midst of a global pandemic, observational studies are associated with inherent limitations such as confounders and biases such as selection and recall bias. Larger patient cohorts and longer-term follow-up are required to confirm the findings as well as ascertain long-term effects on lung cancer mortality.

Another key consideration in managing patients with lung cancers during the COVID-19 pandemic lies in their overlapping clinical and radiological features, which poses unique challenges in the identification of suspect cases of COVID-19 and calls for a high index of suspicion. Overlapping symptoms include cough, dyspnoea, fever, arthralgia/myalgia and fatigue or malaise ${ }^{[9,10,14,15,17,18]}$. Furthermore, immunocompromised lung cancer patients, especially those on myelosuppressive chemotherapy, may present atypically without fever. In addition to clinical symptoms, a detailed history of possible exposure to suspected or confirmed cases of COVID-19 is critical. Patients with thoracic malignancies often have thoracic imaging performed routinely as part of follow-up and response assessment. Chest radiography (CXR) and computed tomography (CT) appearances overlap amongst patients with primary thoracic malignancies, pulmonary metastases, treatment-related pneumonitis (from TKIs, ICIs, or radiotherapy), and COVID-19, with common features such as the presence of unilateral or multifocal ground glass opacities. In COVID-19, ground glass opacities are usually peripheral and/or bibasal. Pulmonary nodules, pleural effusions and mediastinal lymphadenopathy which are seen in thoracic malignancies are less commonly observed in patients with COVID-19 ${ }^{[19]}$. SARS-CoV-2 RT-PCR from nasopharyngeal or respiratory tract (e.g., sputum) samples remains the gold standard for COVID-19 diagnosis.

The unique clinical challenges relating to clinical risk and overlapping presenting features as described above require the use of distinct surveillance protocols from non-cancer patients. On 8 May 2020, the Singapore MOH amended its enhanced Swab-and-Send-Home (SASH) criteria to mandate SARS-CoV2 RT-PCR testing for all cancer patients on chemotherapy presenting with ARI with or without fever. Patients who are not medically unstable nor assessed to require inpatient admission can be managed under the enhanced SASH protocol when they are seen at primary care, instead of being referred to NCID or hospital EDs. Patients with positive results will be recalled and conveyed to NCID. The capacity for diagnostic testing of COVID-19 has been gradually increased nationwide, with expansion from hospitals to Public Health Preparedness Clinics (PHPCs) and polyclinics in the community. From 1 July 2020, the SASH 
criteria were subsequently expanded to include all persons with ARI aged 13 and above in view of increase in community transmission of COVID- 19.

Figure 1 illustrates a management algorithm for cancer patients in TTSH presenting with ARI or radiological findings of pneumonia. For patients on cytotoxic chemotherapy, it is important to exclude secondary infections and neutropenic sepsis which is a life-threatening oncological emergency. Patients with incidental infective changes (not attributable to cancer) noted on routine CXR or CT scans done for response assessment are advised to proceed with COVID-19 testing under the SASH protocol, with nonurgent outpatient appointments postponed to at least 5 days later. Cases where the need for SARS-CoV-2 testing and disposition are unclear are discussed with the COVID-19 consultant on-call. Currently, routine testing of lung cancer patients without fever or ARI before initiation of systemic therapy has not been implemented in our practice.

\section{CHANGE OF PRACTICE IN THE TREATMENT OF LUNG CANCER PATIENTS}

Advances in the pace and scope of research, as well as collaboration across hospitals and oncology centres regionally and worldwide in the creation of multicentre COVID-19 registries, have been critical in providing up-to-date knowledge about COVID-19 and its impact on cancer care and delivery ${ }^{[20]}$. Oncology societies such as the European Society for Medical Oncology (ESMO), National Comprehensive Cancer Network (NCCN) and the International Association for the Study of Lung Cancer (IASLC) have also issued guidelines on lung cancer management during the COVID-19 pandemic ${ }^{[21-23]}$. While providing a relevant overarching framework, it is also important to recognise that variations in healthcare resources and severity of the COVID-19 outbreak across countries affect generalisability of these guidelines. Individualisation of treatment options remains critical.

The massive rise of COVID-19 infections in Singapore has caused an overload on the healthcare system and resources have to be reprioritised and distributed between patients with COVID-19 infections as well as to continue ongoing medical care to the rest of the patient population. Healthcare workers are also under the pump as clinic staff, nurses and doctors get out-posted to the COVID-19 frontline, leaving less team members available to continue with the routine clinical workload. During the pandemic, many elective surgeries and non-urgent procedures are deferred; however, disciplines such as the medical oncology and radiation oncology departments often could not do the same. It is crucial for patients with lung cancer to continue treatments in a timely fashion despite the pandemic. The number of patients seen at the oncology outpatient clinic at TTSH in Singapore has also remained relatively stable during the DORSCON Orange pandemic period despite a likely reduction in the number of patients undergoing investigations for new cancer diagnoses and less elective surgeries. The average monthly number of specialist outpatient clinic attendances in our hospital for patients with newly diagnosed thoracic malignancies increased slightly by $10 \%$ from 2019 to 2020 , suggesting the absence of treatment delays despite the pandemic for patients with newly diagnosed thoracic malignancies requiring systemic therapy. The number of followup visits decreased slightly by approximately $8 \%$, likely due to the planned postponement of non-urgent appointments such as for patients on survivorship follow-up [Table 1].

There were a total of 1,356 outpatient visits for patients with thoracic malignancies in TTSH from January to August 2020. There were only 2 cases of COVID-19 diagnosed amongst these patients, both community acquired without evidence of nosocomial transmission, and zero cases in medical and nursing staff within the medical oncology department.

Despite the potential increased risk of inadvertent COVID-19 exposure, it is important to weigh the competing risks of disease progression and treat the patients urgently especially in the cases where there is an opportunity for cure. The ESMO guidelines regarding treatment of cancer patients during the 
Table 1. Specialist outpatient clinic attendances for thoracic cancers in TTSH in 2019 and 2020, prior to and during the COVID-19 pandemic

\begin{tabular}{llccc}
\hline & & 2019 (Jan-Dec) & 2020 (Jan-Aug) & Percentage change \\
\hline No. of first visits & Total & 135 & 100 & $+10 \%$ \\
& Monthly average & 11.25 & 12.5 & \\
No. of review visits & Total & 2,048 & 1,256 & $-8 \%$ \\
& Monthly average & 170 & 157 & \\
\hline
\end{tabular}

TTSH: Tan Tock Seng Hospital; COVID-19:Coronavirus disease 2019; No.: number

pandemic also stated that high priority should be given for outpatient lung cancer patient with a new diagnosis or suspicion of invasive lung cancer (clinical stage II/IIIA/IIIB or metastatic NSCLC or SCLC) and patients with disease-related symptoms (dyspnoea, pain, haemoptysis, etc.) and the need for visits for treatment administration ${ }^{[24]}$. Recently, many guidelines and consensus statements have been published related to cancer management during the COVID-19 pandemic to provide guidance to oncologists during this difficult period ${ }^{[2,23,25]}$. The guidelines recommend that the clinical situation and quality of care for a patient with lung cancer should not be changed, treatment plans should continue to be discussed with a multidisciplinary team and implementation of the recommendations by the multidisciplinary team should not be considered negotiable in light of the COVID-19 pandemic and its challenges ${ }^{[26]}$. The ESMO guidelines are defined by 3 levels of therapeutic intervention. Firstly, the high priority tier focuses on immediately life-threatening cases, clinically unstable cases, and/or cases where the magnitude of benefit appears to be substantial leading to a significant improvement in overall survival or quality of life (QOL). Medium priority cases include non-critical situations where any more than a 6- to 8-week delay could impact the patient's overall outcome or magnitude of benefit. The lowest priority tier is defined as stable conditions that allow services to be delayed for the duration of the pandemic with no survival benefit or a change/reduction in QOL ${ }^{[25,26]}$. Lung cancer treatment at TTSH follows the guidelines as specified by ESMO in a similar fashion and adapts a "business as usual" model for patients requiring high priority treatment [Table 2].

For patients with stage III lung cancer who require definitive chemoradiation and small cell lung cancer patients who require urgent systemic chemotherapy, the medical oncology department at TTSH continues to prioritise timely interventions and strives to provide the same quality of care as in pre-pandemic situations. Lung multidisciplinary meetings have continued to function on a regular basis discussing complex cases with provisions for the setup of adequate social distancing and ensuring only attendance of key personnel during this period of time to reduce exposure risk and to segregate teams as mentioned previously.

For patients with small cell lung cancer where systemic chemotherapy is known to be effective and timesensitive, medical oncologists often still opt to institute chemotherapy urgently and the first cycle can continue to be given as an inpatient. Patients who are diagnosed in the peripheral hospitals who require urgent treatment and medical oncology management would be reviewed on a case-by-case basis and transfer to TTSH can be arranged upon approval by the Head of Department, Division Head and the Chairman of the Medical Board of both hospitals.

The medical oncology department at TTSH has instituted certain measures to review the outpatient clinic list with aims to postpone appointments for patients deemed feasible for a longer interval review (those with low/intermediate risks of relapse) or who are on mainly survivorship follow-ups. The primary oncologist in charge of the clinic is asked to review the list of patients scheduled for outpatient clinics during the height of the pandemic period and prescriptions may be topped up for patients on targeted therapies if they are deemed to be stable and may be able to delay their follow up appointments. A 
Table 2. Priorities of treatment for lung cancer patients at TTSH (Adapted from ESMO guidelines of management of lung cancer patients during the COVID-19 pandemic)

\begin{tabular}{lll}
\hline Small cell lung cancer treatment & $\begin{array}{c}\text { Prioritised without } \\
\text { delay }\end{array}$ & $\begin{array}{c}\text { Lower priority/ } \\
\text { Delay appointment }\end{array}$ \\
Neoadjuvant chemotherapy in clinical stage III NSCLC & $\star$ \\
Concomitant or sequential chemoradiotherapy for inoperable stage III & $*$ \\
NSCLC & $*$ \\
Starting consolidation durvalumab (within 42 days) & \\
Delivery of adjuvant chemotherapy in stage II/III as recommended by \\
lung tumour board \\
Newly diagnosed lung cancer patients with targetable mutations \\
Newly diagnosed lung cancer patients who are candidates for 1st-line \\
treatment including chemotherapy, chemotherapy plus IO, IO alone or \\
TKIs to improve prognosis, cancer-related symptoms and QOL \\
Start 2nd-line chemotherapy or IO in symptomatic and progressive \\
disease patients \\
Start 2nd-line TKI in progressive disease patients \\
Oncological emergencies (management of hypercalcaemia, cord \\
compression, SVC obstruction, serious immune mediated adverse \\
effects etc.) \\
Start 2nd and beyond line chemotherapy or IO in asymptomatic \\
patients, in absence of threatening disease (volume/location) \\
Discussion of adjuvant chemotherapy for Stage IB \\
Anti-PD-(L)1 scheduled cycles may be modified/delayed to reduce \\
clinical visits (for instance, using 4-weekly or 6-weekly dosing instead \\
of 2- or 3-weekly for selected agents when appropriate) \\
Postpone antiresorptive therapy (zoledronic acid, denosumab) that is \\
not needed urgently \\
Follow-up for patients at low/intermediate risk of relapse \\
Survivorship visits \\
\hline
\end{tabular}

TTSH: Tan Tock Seng Hospital; ESMO: European Society for Medical Oncology; COVID-19: Coronavirus disease 2019; NSCLC: nonsmall cell lung cancer; TKIs: tyrosine kinase inhibitors; IO: ImmunoOncology drug; QOL: quality of life; SVC: superior vena cava; PD-(L)1: Programmed death-(ligand) 1

medication delivery service has been set up by the hospital pharmacy which provided convenience to patients and carers during the pandemic period in avoiding hospital visits and reducing long wait times at the outpatient pharmacies. Without compromising patient safety and efficacy, systemic treatment regimens should be adjusted to reduce hospital visits. Immunotherapy treatments may be given with a longer interval via dose adjustments such as Nivolumab at $480 \mathrm{mg}$ 4-weekly instead of $240 \mathrm{mg}$ fortnightly; and $400 \mathrm{mg}$ Pembrolizumab 6-weekly instead of $200 \mathrm{mg}$ 3-weekly. Efforts are also currently underway for pilot and subsequent larger scale implementation of telemedicine consults.

Important clinical trials that are deemed to impact the patient's overall outcome and have a significant magnitude of benefit were able to continue treatment throughout the entire DORSCON Orange period. Trials that may not have a significant impact on patient's outcomes, in particular, qualitative research and survey studies were placed on hold for recruitment during the height of the pandemic period as well.

\section{EDUCATION AND TRAINING OF RESIDENTS AND JUNIORS DURING COVID-19 PANDEMIC}

The COVID-19 pandemic has led to a significant impact on post-graduate education in Singapore and educators now need to consider alternative novel methods of providing education to minimise disruption to training of the junior medical staff ${ }^{[27]}$. Almost universally across all academic centres, there has been a transition to teaching via videoconferencing technologies which has been recognized as an effective teaching modality in situations where distant learning is required ${ }^{[2,29]}$. However, online videoconferencing teaching had posed several issues including the need for additional information technology resources and funding for extra laptops, projectors and speakerphones. There is also a need to ensure that the students have access to a stable internet connection throughout the different sites during the online teaching 
sessions. Educators also need to adapt to these new practices and come up with effective teaching strategies for web conferencing, which can be challenging for physicians who are used to conventional bedside teaching of students and trainees ${ }^{[29]}$.

To reduce the risks of inter- and intra-departmental infection transmission, different meeting rooms are also utilized to segregate the inpatient and outpatient service teams during the videoconferencing teaching sessions or multidisciplinary case discussions. The weekly scheduled medical oncology teaching sessions at TTSH utilized pre-recorded lectures on various subspecialty topics supervised by a consultant in charge. Bedside teaching during ward rounds to the inpatient junior team would be led by the ward consultant in charge. Case discussions and clinical handover to the weekend on-call team using third party software and online platforms also posed concerns in ensuring security of information in terms of the Personal Data Protection Act (PDPA), which came into full effect in July 2014 in Singapore.

During the DORSCON Orange alert period, there is a need to minimise cross cluster transfer of medical staff, hence rotations of residents and registrars could only be limited to the current institution that they are based in with no external rotations to other hospitals allowed. Although this may lead to a certain level of disruption to the training exposure, measures are in place to ensure that they are allowed to be rotated to a different department of choice within the same academic institution.

For staff deployed to the NCID to manage outbreak wards, timely training is conducted to refresh their knowledge on Personal Protective Equipment (PPE) and to orientate them to the facilities and equipment at NCID. Communications on the latest developments of the COVID-19 situation and updated protocols on screening and managment for COVID-19 cases are available on the hospital's intranet and distributed electronically to staff via emails.

It is critical to provide emotional support to clinical staff and trainees during this difficult period given the high risk of burnout when faced with such a prolonged crisis. A recent survey was conducted by the NHG residency office pertaining to the challenges faced by residents as a result of the interruptions to training posed by the ongoing COVID-19 outbreak. They reported an increase in the level of stress and burnout, citing an average of 4.7 on a scale of 0 (no stress at all) to 10 (extreme level of stress) and $61 \%$ of the respondents either agree or strongly agree when asked whether the current outbreak had adversely affected their training and or career ${ }^{[30]}$. TTSH has set up the 3S (Staff Support Staff) hotline with the ICU(Intensive Care Unit) teams providing an emotional debrief session and also a technical debrief to iron out any problems faced at each shift. The Staff Wellbeing Committee is also set up to ensure that staff feels supported and to ensure meals and snacks are distributed to the staff on duty in the outbreak wards.

\section{VACCINE DEVELOPMENT IN CLINICAL TRIAL EVALUATION}

It is a race against time and a global effort is made towards rapid SARS-CoV-2 vaccine development with the aim of inducing protective immunity following vaccination. There are now phase II clinical trials with a trajectory towards phase III efficacy evaluation, however there are added challenges with the dynamic changes seen with this virus ${ }^{[31-33]}$.

Patients with advanced cancer have historically been under-represented in vaccine clinical trials. As targeted therapy and immunotherapy have significantly improved the life expectancy for patients with metastatic NSCLC, these patients should not be reflexively excluded from vaccine trials on the basis of old preconceptions limiting care for these patients. We advocate that vaccine trials should include patients with malignancies including thoracic cancers, so as to ascertain safety and efficacy in this group of patients. 


\section{CONCLUSION}

Compared to prior pandemics, we now have far greater scientific, medical and technological capabilities to manage COVID-19, but also increased vulnerabilities and transmission risks in a globalised world and economy. COVID-19 has had wide-ranging implications globally, ranging from its impact on healthcare systems to economic and long-term psychological impact. Infection control and outbreak mitigation measures, and the availability of effective therapeutics and vaccines will shape the eventual outcome of the pandemic.

Oncologists have a special duty to our patients to ensure patient safety and provide optimum care without undue disruption which may compromise long-term cancer-specific outcomes. We must carefully weigh the management of an established threat (lung cancer) where treatment delays can lead to worse outcomes, versus the uncertain additional risk of COVID-19 infection. Treatment decisions should be individualised based on best available evidence, along with multidisciplinary input and shared decision-making with patients. Important areas for future research include long term COVID-19 and cancer-specific outcomes, late complications and survivorship issues, and psychological impact on patients and healthcare providers.

The COVID-19 pandemic has significantly changed day-to-day practices within our institution. Singapore has been relatively fortunate in having a structured and well-prepared healthcare system to cope with the COVID-19 pandemic, with ready accessibility to SARS-CoV-2 testing, good population compliance to public health measures and appropriate reallocation of healthcare resources without excessive disruption to the care of oncology patients. With better control of the outbreak locally, there has also been a corresponding gradual increase in the provision of routine oncology services back to pre-COVID levels. We must continue to adapt to the evolving situation to ensure preservation of healthcare capacity, protection of vulnerable populations and care of oncology patients in the form of a sustainable new normal for the future.

\section{DECLARATIONS}

Authors' contributions

Conception, manuscript writing, final approval of manuscript: Vong EKY, Chia PL, Chang AY

\section{Availability of data and materials}

Not applicable.

\section{Financial support and sponsorship}

All authors have declared that they have no financial relationships at present that is related to this paper.

\section{Conflicts of interest}

All authors declared that there are no conflicts of interest.

\section{Ethical approval and consent to participate}

Not applicable.

\section{Consent for publication}

Not applicable.

\section{Copyright}

(c) The Author(s) 2020.

\section{REFERENCES}

1. Johns HOpkins UNiversity and Medicine coronavirus resource centre mortality analyses 2020. Available from: https://coronavirus.jhu. edu/data/mortality. [Last accessed on 8 Dec 2020] 
2. Beigel JH, Tomashek KM, Dodd LE, et al. Remdesivir for the treatment of Covid-19- preliminary report. N Engl J Med 2020;383:994.

3. Group RC, Horby P, Lim WS, et al. Dexamethasone in hospitalised patients with Covid-19 - preliminary report. $N$ Engl J Med 2020; NEJMoa2021436.

4. gov.sg. What do the different DORSCON levels mean 2020. Available from: https://www.gov.sg/article/what-do-the-different-dorsconlevels-mean. [Last accessed on 8 Dec 2020]

5. National centre for infectious diseases (NCID). About NCID. Available from: https://www.ncid.sg/About-NCID/Pages/default.aspx. [Last accessed on 8 Dec 2020]

6. Urun Y, Hussain SA, Bakouny Z, et al. Survey of the impact of COVID-19 on Oncologists' decision making in cancer. JCO Glob Oncol 2020;6:1248-57.

7. London JW, Fazio-Eynullayeva E, Palchuk MB, Sankey P, McNair C. Effects of the COVID-19 pandemic on cancer-related patient encounters. JCO Clin Cancer Inform 2020;4:657-65.

8. Maringe C, Spicer J, Morris M, et al. The impact of the COVID-19 pandemic on cancer deaths due to delays in diagnosis in England, UK: a national, population-based, modelling study. Lancet Oncol 2020;21:1023-34.

9. Report of the WHO-China joint mission on coronavirus disease 2019 (COVID-19) 2020. Available from: https:/www.who.int/docs/ default-source/coronaviruse/who-china-joint-mission-on-covid-19-final-report.pdf. [Last accessed on 8 Dec 2020]

10. Wu ZY, McGoogan JM. Characteristics of and important lessons from the coronavirus disease 2019 (COVID-19) outbreak in China: summary of a report of 72314 cases from the Chinese Center for Disease Control and Prevention. JAMA 2020;323:1239-42.

11. National University Cancer Institute of Singapore Workflow T. A segregated-team model to maintain cancer care during the COVID-19 outbreak at an academic center in Singapore. Ann Oncol 2020;31:840-3.

12. Tan BF, Tuan JKL, Yap SP, Ho SZ, Wang MLC. Managing the COVID-19 pandemic as a national radiation oncology centre in Singapore. Clin Oncol (R Coll Radiol) 2020;32:e155-9.

13. Desai A, Sachdeva S, Parekh T, Desai R. COVID-19 and cancer: lessons fom a pooled meta-analysis. JCO Glob Oncol 2020;6:557-9.

14. Kuderer NM, Choueiri TK, Shah DP, et al. Clinical impact of COVID-19 on patients with cancer (CCC19): a cohort study. Lancet 2020;395:1907-18.

15. Lee LYW, Cazier JB, Starkey T, et al. COVID-19 mortality in patients with cancer on chemotherapy or other anticancer treatments: a prospective cohort study. Lancet 2020;395:1919-26.

16. Solodky ML, Galvez C, Russias B, et al. Lower detection rates of SARS-COV2 antibodies in cancer patients versus health care workers after symptomatic COVID-19. Ann Oncol 2020;31:1087-8.

17. Garassino MC, Whisenant JG, Huang LC, et al. COVID-19 in patients with thoracic malignancies (TERAVOLT): first results of an international, registry-based, cohort study. Lancet Oncol 2020;21:914-22.

18. Luo J, Rizvi H, Preeshagul IR, et al. COVID-19 in patients with lung cancer. Ann Oncol 2020;31:1386-96.

19. Shi HS, Han XY, Jiang NC, et al. Radiological findings from 81 patients with COVID-19 pneumonia in Wuhan, China: a descriptive study. Lancet Infect Dis 2020;20:425-34.

20. Guckenberger M, Belka C, Bezjak A, et al. Practice recommendations for lung cancer radiotherapy during the COVID-19 pandemic: an ESTRO-ASTRO consensus statement. Radiother Oncol 2020;146:223-9.

21. Dingemans AC, Soo RA, Jazieh AR, et al. Treatment guidance for patients with lung cancer during the coronavirus 2019 pandemic. $J$ Thorac Oncol 2020;15:1119-36.

22. National Comprehensive Cancer Network. Short-term recommendations for non-small cell lung cancer management during the COVID-19 pandemic. JCO Oncology Practice 2020;16:579-86.

23. Curigliano G, Banerjee S, Cervantes A, et al. Managing cancer patients during the COVID-19 pandemic: an ESMO interdisciplinary expert consensus. Ann Oncol 2020;31:1320-35.

24. ESMO. ESMO management and treatment adapted recommendations in the COVID-19 era: lung cancer 2020 16/08/2020. Available from: https://www.esmo.org/guidelines/cancer-patient-management-during-the-covid-19-pandemic/lung-cancer-in-the-covid-19-era. [Last accessed on 8 Dec 2020]

25. Passaro A, Addeo A, Von Garnier C, et al. ESMO management and treatment adapted recommendations in the COVID-19 era: lung cancer. ESMO Open 2020;5:e000820.

26. Ternyila D. Recommendations for managing patients with lung cancer during COVID-19 Era: Targeted Oncology; 2020 Available from: https://www.targetedonc.com/view/recommendations-for-managing-patients-with-lung-cancer-during-covid-19-era. [Last accessed on 8 Dec 2020]

27. Sia CH, Tan BY, Ooi SBS. Impact of the coronavirus disease 2019 pandemic on postgraduate medical education in a Singaporean academic medical institution. Korean J Med Educ 2020;32:97-100.

28. Sidpra J, Gaier C, Reddy N, Kumar N, Mirsky D, Mankad K. Sustaining education in the age of COVID-19: a survey of synchronous web-based platforms. Quant Imaging Med Surg 2020;10:1422-7.

29. Byrnes KG, Kiely PA, Dunne CP, McDermott KW, Coffey JC. Communication, collaboration and contagion: "Virtualisation" of anatomy during COVID-19. Clin Anat 2020;34:82-89.

30. Wong CS, Tay WC, Hap XF, Chia FL. Love in the time of coronavirus: training and service during COVID-19. Singapore Med J 2020;61:384-6.

31. Corbett KS, Edwards D, Leist SR, et al. SARS-CoV-2 mRNA vaccine development enabled by prototype pathogen preparedness. bioRxiv $2020 ; 145920$.

32. Jackson LA, Anderson EJ, Rouphael NG, et al. An mRNA vaccine against SARS-CoV-2 - preliminary report. $N$ Engl J Med 2020;383:1920-31.

33. Tai WB, Zhang XJ, Drelich A, et al. A novel receptor-binding domain (RBD)-based mRNA vaccine against SARS-CoV-2. Cell Res 2020;30:932-935. 